\title{
Scene Analysis and Individual Tracking through Multiple Cameras
}

\author{
Priya Singh \\ B-Tech in Computer Science \& Engineering \\ $\mathrm{M}$-Tech in Computer Science \& Engineering
}

\begin{abstract}
Scene Analysis and Individual Tracking through Multiple Camera (SAITMC) is a complex task that generally requires the use of different analysis for specific purposes. This involve basically the analysis of background using specific method and after that the tracking of particular individual is taken place from firstly through a single camera and then the same procedure is taken over the multiple camera. There are many advantages of doing this as it will provide a very efficient way through which we can track the individual at various public places like in a mall, in a building etc. SAITMC helps to perform the background analysis and then perform the tracking of individual through single camera and then the same through the multiple cameras.
\end{abstract}

\section{Keywords}

Scene Analysis, Individual Tracking, Multiple Cameras.

\section{INTRODUCTION}

Scene Analysis and Individual Tracking through Multiple Camera (SAITMC) is an important task within the field of computer vision. The rapid advancement of high-powered computers, the availability of better quality, affordable and reasonable video cameras, and the raise in need for automated video analysis has generated a great deal of interest in individual tracking algorithms. The main intension of individual object detection and tracking is to establish a link between individual parts of object in consecutive frames and to retrieve its information such as posture and gesture of the particular one. Tracking detected objects frame by frame in video is a difficult task. It is a critical part of smart surveillance systems since without object tracking, the system could not retrieve unite full information about individuals and higher level activity analysis which involve multiple steps.

\section{PROBLEM \& NEED DEFINITIONS}

This paper includes the way through which we can detect and track multiple objects through multiple cameras. It includes firstly object detection or individual detection then tracking that object in a whole video and providing a unique identifier to that object. After that, this information is sent to other cameras so that the same object can be treated with the same identifier given earlier. With this, we can track an object from its time of entry to its exit in the region of space covered by the camera network.. There are many benefits of doing this basically in the field of security, video surveillance etc. Tracking individual object is of interest for a variety of complex applications such as surveillance, activity monitoring, etc. How to efficiently track moving targets in the observation scope has become an important issue. As a result, systems having efficient tracking results need to be introduced. To cover an area of interest, it is reasonable to use cameras with overlapping field of views.

\section{KEY FUNCTIONS}

\section{A. Non Recursive}

A Non Recursive technique uses a sliding window approach for background estimation. Non recursive techniques are highly adaptive as they do not depend on the history beyond those frames stored in the buffer. Some of the techniques are:

1) Frame differencing-

The simplest way to implement this is to take an image as background and take the frames obtained at the time t, denoted by $\mathrm{I}(\mathrm{t})$ to compare with the background image denoted by $\mathrm{B}(\mathrm{t})$. In mathematical equation, it is written as:

$$
\begin{aligned}
& \mathbf{F}(\mathbf{t})=\mathbf{I}(\mathbf{t})-\mathbf{B}(\mathbf{t}) \\
& \mathbf{F}(\mathbf{t})-\mathbf{F}(\mathbf{t}-\mathbf{1})>\mathbf{T h}
\end{aligned}
$$

Where $\mathrm{F}(\mathrm{t}-1)$ is the previous image. And Th is defined threshold.

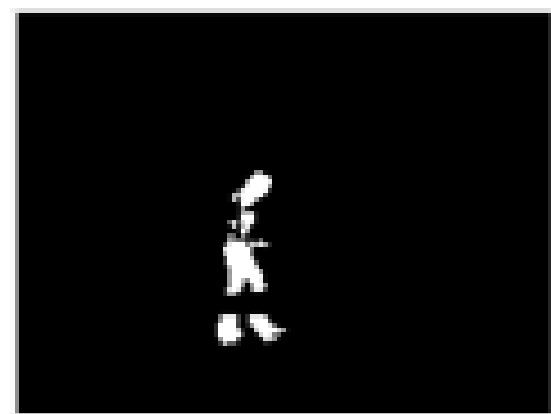

2) Median filtering

In median filtering, the previous $\mathrm{N}$ frames of video are buffered, and the background is calculated as the median of buffered frames. Then same as with frame difference the background is subtracted from the current frame and apply threshold to determine the foreground pixels.

$B(x, y, t)=\frac{1}{N} \sum_{i=1}^{N} V(x, y, t-i)$

where $\mathrm{N}$ is the number of preceding images taken for averaging. After calculating the background $\mathrm{B}(\mathrm{x}, \mathrm{y}, \mathrm{t})$, we can then subtract it from the image $\mathrm{V}(\mathrm{x}, \mathrm{y}, \mathrm{t})$ at time $t=\mathrm{t}$ and threshold it. Thus the foreground is

$$
\mathbf{V}(\mathbf{x}, \mathbf{y}, \mathbf{t})-\mathbf{B}(\mathbf{x}, \mathbf{y}, \mathbf{t})>\mathbf{T h}
$$

Where Th is threshold. 


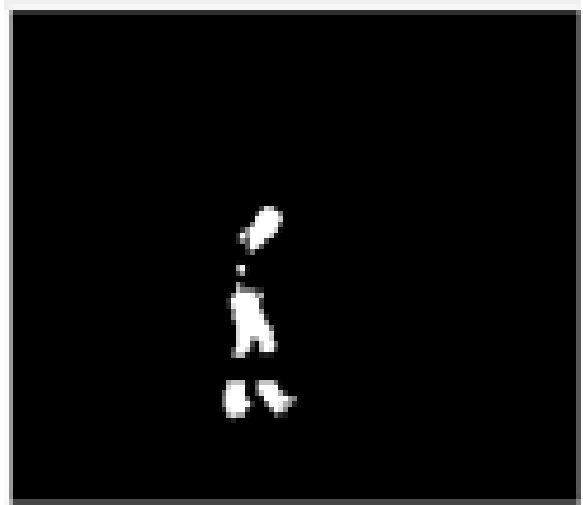

3)Mean filtering - it is similar to the median filtering but the only difference is we take the mean of all the images instead of taking median.

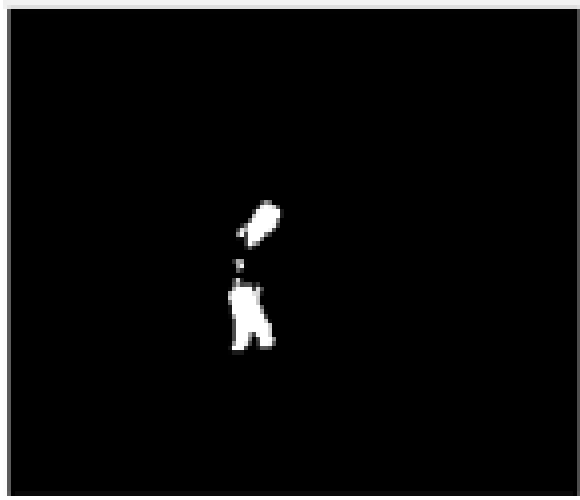

\section{B. Recursive}

They do not maintain buffer for background modelling. It involve below methods:

\section{1) Single Gaussian:-}

In this we have taken the mean of some initial frames and store them as a background $\mathrm{B}(\mathrm{x}, \mathrm{y}, \mathrm{t})$ and then subtract every new frame $\mathrm{D}(\mathrm{x}, \mathrm{y}, \mathrm{t})$ with this background but here we are not comparing with the threshold value but with new variable which is multiple of some constant value $\mathrm{K}$ with standard deviation $\operatorname{SD}(x, y, t)$ and if the difference between background and frame value is greater than $\mathrm{S}(\mathrm{x}, \mathrm{y}, \mathrm{t})$ then its background else foreground.

$$
\begin{aligned}
& \mathbf{R}(\mathbf{x}, \mathbf{y}, \mathbf{t})=\mathbf{B}(\mathbf{x}, \mathbf{y}, \mathbf{t})-\mathbf{D}(\mathbf{x}, \mathbf{y}, \mathbf{t}) \\
& \mathbf{S}(\mathbf{x}, \mathbf{y}, \mathbf{t})=\mathbf{K} * \operatorname{SD}(\mathbf{x}, \mathbf{y}, \mathbf{t}) \\
& \text { If } \mathbf{R}(\mathbf{x}, \mathbf{y}, \mathbf{t})>\mathbf{S}(\mathbf{x}, \mathbf{y}, \mathbf{t})
\end{aligned}
$$$$
F(x, y, t)=1 \quad \text { as background }
$$

Else

$$
\mathbf{F}(\mathbf{x}, \mathbf{y}, \mathbf{t})=\mathbf{0} \quad \text { as foreground }
$$

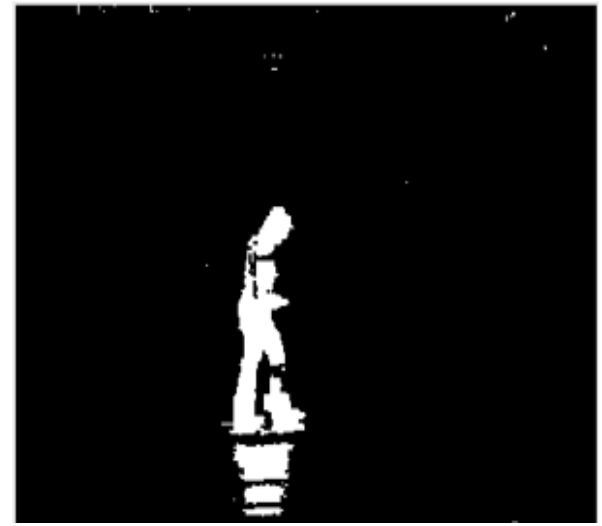

Among the above single Gaussian gives the perfect result so we are using single Gaussian method.

\section{Morphological Operations (MO)}

Morphology, it is a broad class of image processing and computer vision that process or compute the image based on their properties such as their color, shape. It perform all the computation and operations on the object based on their features.

And to perform this, two main morphological operations are used that are Erosion and Dilation. Morphological operators such as Erosion and Dilation generates an output image of the same size by applying the structuring element on the input image or frame.

Erosion : Use to remove pixels from the boundary of image.

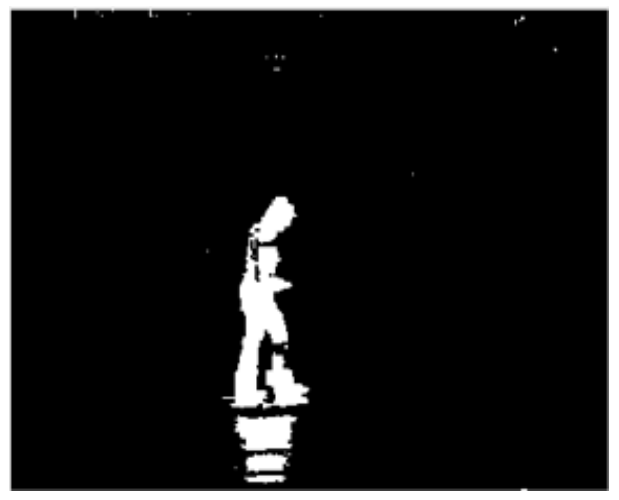

Dilation: Use to add pixels in the boundary of image.

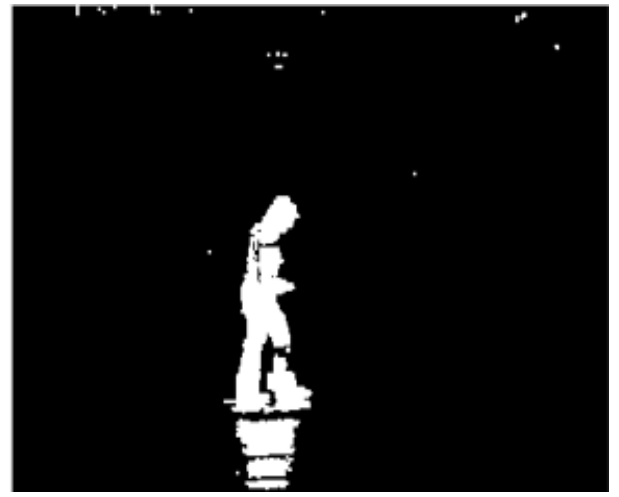

\section{Masking}

Mask is a filter and also known as spatial filtering. In this we are simply doing the filtering operation that are performed directly on the image. A mask is a small matrix whose values are called weights.. We have used the masking after doing the background subtraction. The output of background subtraction 
is a binary image that contain the object with the white color and the background with the black color. And after doing the masking what we are actually doing is putting the original frame on the background subtracted frame which result into a frame with the real object placed with a background to be black.

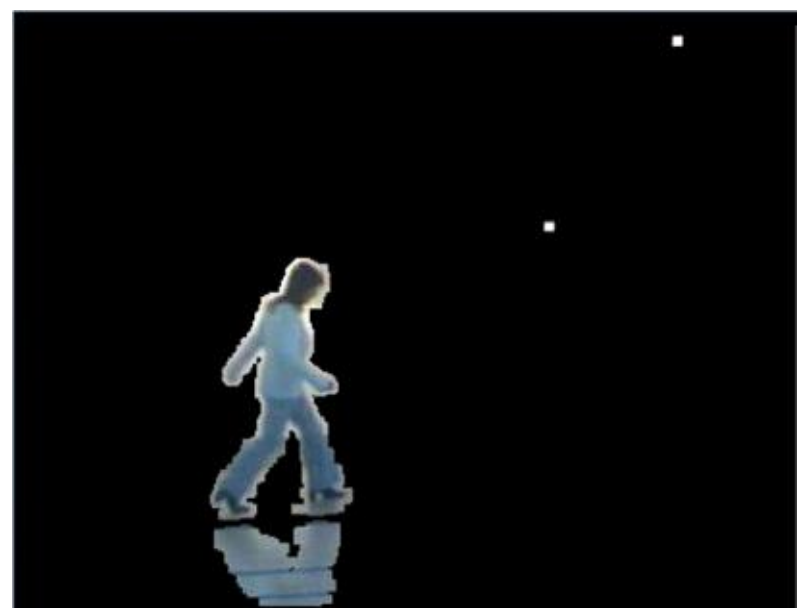

E. Regionprops

It is the function that is used to measure or calculate the properties of image regions of input image that contain the object and perform the desired operation and computation on them.

\section{$\mathrm{S}=$ regionprops $(\mathrm{B}$, properties $)$}

$\mathrm{B}=$ Binary Image

Properties $=$ Define the specific property of binary image

Here we are using bounding box property to create rectangular region along the object in a frame.

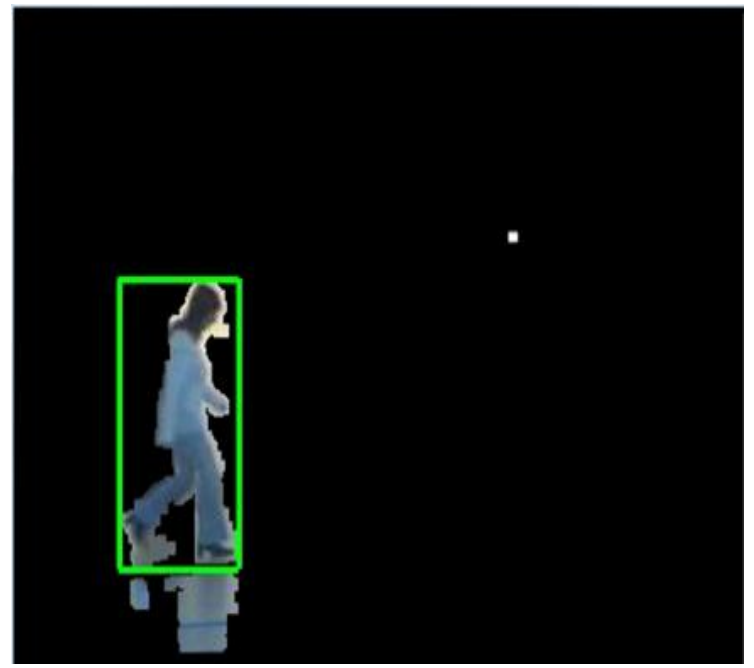

\section{F. Histogram}

This is used to find the histogram of image data. And the syntax is given as:

$\mathrm{P}=\operatorname{imhist}(\mathrm{I})$;

Imhist (I) is used to calculate the histogram of image given as $\mathrm{I}(\mathrm{x}, \mathrm{y})$ and plot a histogram for the object

For displaying the histogram of image we can also use :

$\mathbf{P}=$ hist (I)
hist(I) displays a histogram of the element in vector I. The element in I are sorted into ten equally spaced bins along the $\mathrm{x}$-axis between the maximum and minimum value of $\mathrm{I}$ and it displays the bins in the form of rectangle, such that the height of each rectangular bin shows the number of element in the bin.

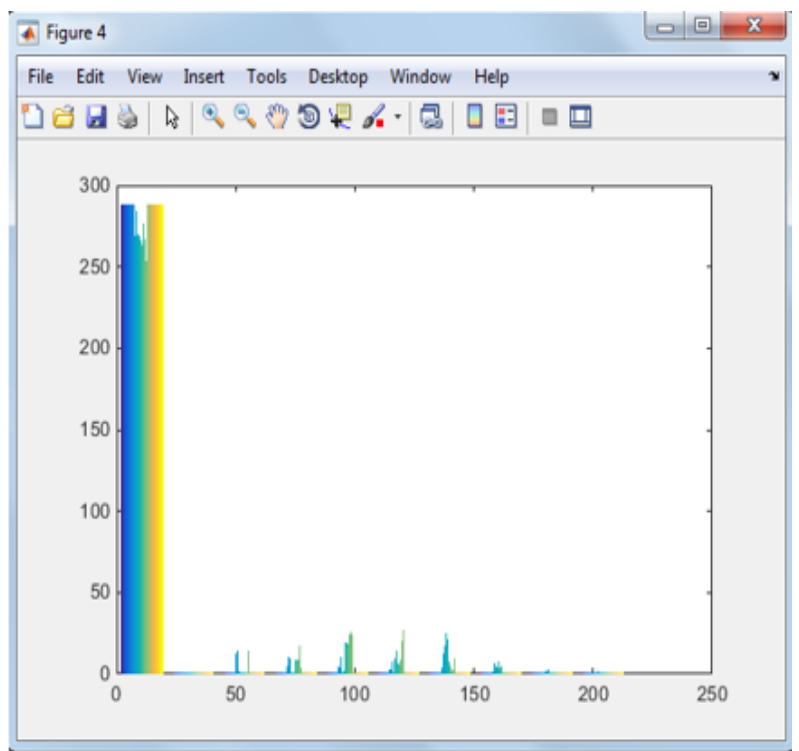

\section{G. Mean Shift Tracking}

It is the algorithm that is used in tracking object and that works by iteratively shift a data point to the mean or average of all the near by data points. It is similar to clustering and have many application areas in the field of tracking, clustering, probability density estimation etc. It is approach for tracking multiple object in multiple frames or in a whole video in which the centroid of object are assumed to be the central part are proposed. It is also the histogram based target representation by kernel. The target localization and feature information is not sufficient for enhance localization so some structure information is added. MST finds the area of a video frame that is locally most similar to the previously defined model. The selected image region has to be tracked in whole video by creating the histogram of that particular region.

\section{H. Single Object through Multiple Camera}

In this we will first detect the object to track by creating the bounding box and then it store the color histogram of the object detected in bounding box and start tracking the same object from one camera and at the same time the stored information being transferred to another camera and the same object is detected in another camera. This is also done with the help of mean shift tracking and we use the extension of mean shift algorithm on multiple camera. In this section we do the tracking of single object through multiple camera. 


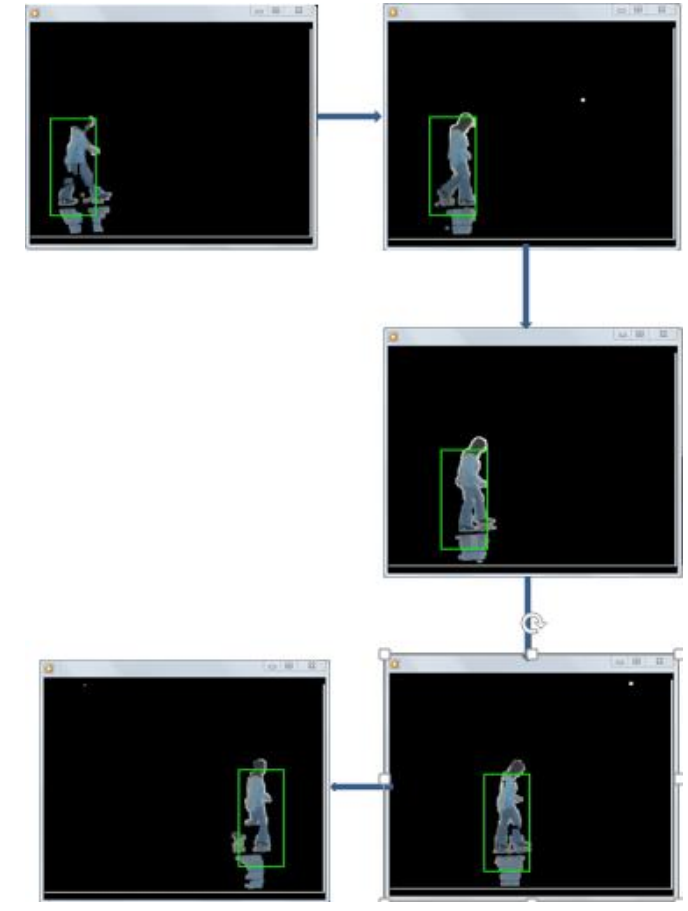

\section{Multiple Object through Multiple Camera}

In this we will first detect the multiple object to track by creating the bounding box along them and then it store the color histogram of all the object detected in bounding box and start tracking the same object from one camera and at the same time the stored information being transferred to another camera and the same object is detected in another camera and this is being done on all the objects that are detected at the same time.

\section{Camera 2: Corridor view}

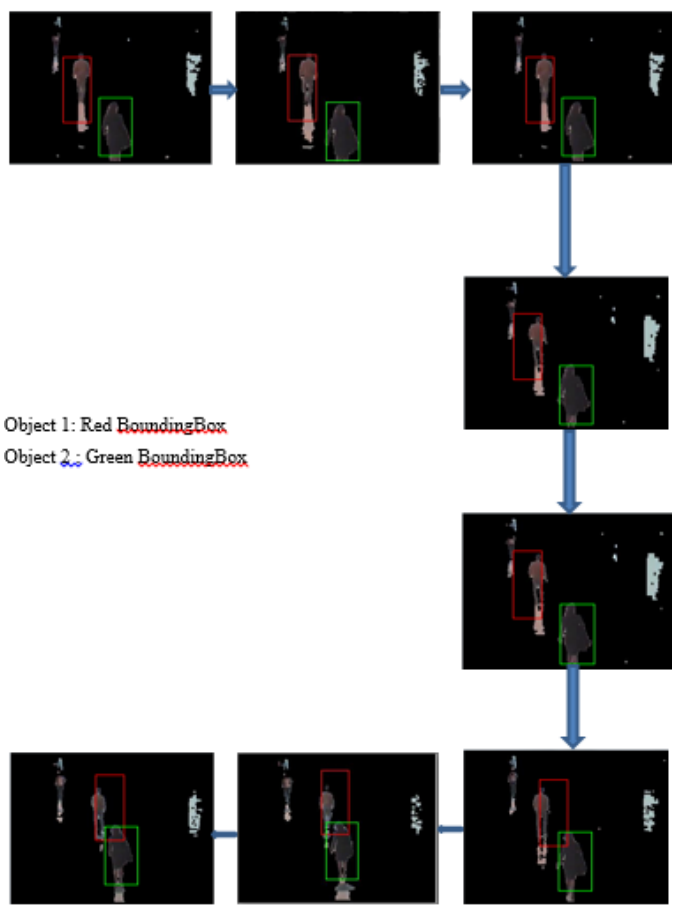

Camera2: Front view

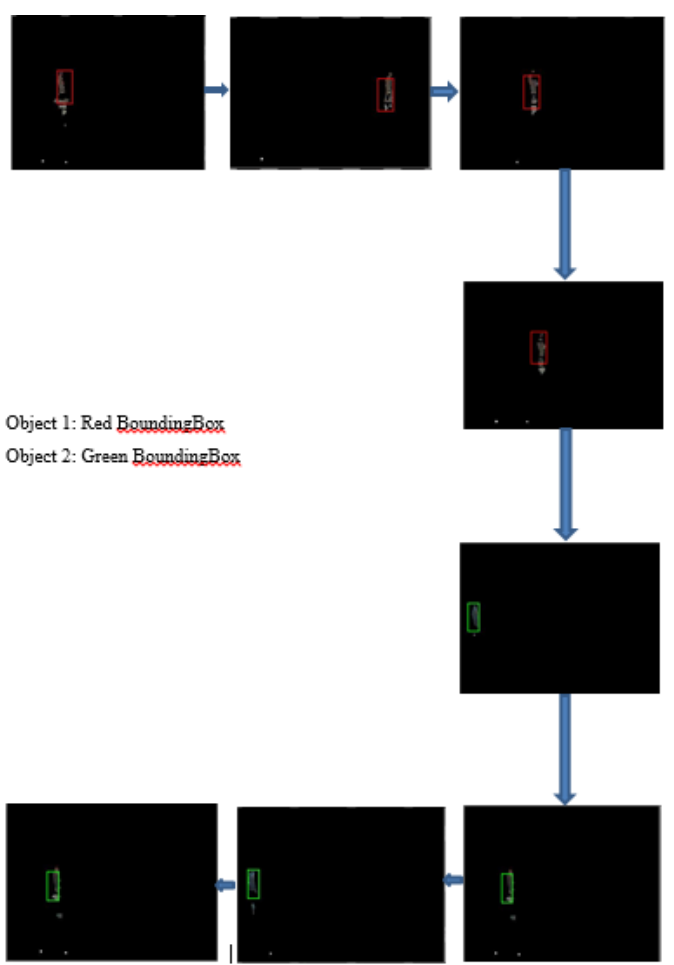

\section{HARDWARE, \& Software Involved}

A. Use of Device Hardware

It combines with all components like desktop/laptop, Battery, and charger with external video to be tested as well as the database to be required and the necessary parts. All parts are assemble in compact device.

\section{B. Use of Tracking software}

Tracking software use to track the object and spot the current location and act immediately when anyone needs emergency support. On a object tracking system the output come only in video format, object name with its unique color id. This whole thing is done on MATLAB.

\section{PURPOSED FLOWCHART \& ALGORITHM} Step 0 -

Initially we need a video on which the objects are given. Firstly we will start with background analysis or scene analysis.

Step 1 -

For performing the scene analysis we perform many methods of recursive and non recursive method and select the best one to detect object. As we have choose Single Gaussian method. The output of this method is shown earlier which contains the object in white pixel and the background comes as the black one..

Step 2 -

Till now we have the output in white and black pixel form, the object consist of white and the background consist of black but for removing noise in object we perform the morphological operations on them that are dilation and erosion. Dilation are used to add pixel in the frame and the erosion is used to remove pixels from the frame. And the output of both are shown earlier. 
Step 3 -

After doing the morphological operations, we perform the masking. By doing the masking we will get the white portion of the object to be covered with the object's actual gesture. Like wise the background is still black but the white pixel area include the actual value of pixel that are present in video. And it is shown above how object is masked with its actual gesture but the background remain unchanged.

Step 4 -

Now we get the object in a frame so the main thing comes to track the object for that we need to use the regionprops function and create the unique color boundingbox along the object. As it allocate red color to some object, green to another and so on using bounding box properties.

Step 5 -

After creating the bounding box we have to track the object so we use mean shift tracking. MST select the object first and then the same object is being tracked in a whole video by comparing the histogram of the object and creates the unique color boundingbox along the object. Mean shift tracker tracks the object by simply taking the object in first frame and create the histogram for that than again takes the same object in next frame and creates the histogram of the same and then compare histogram of both to check whether they are same or not till the object is shown in a video the compared histogram is approximately same otherwise it differ. And tracker understand that this is another object.

\section{Step 6 -}

When the object is tracked through one camera then the same object detected histogram being given to the videos taken from other cameras and if the same object is detected in other camera view then it automatically create the boundingbox with the same color that was given earlier.

Step 7 -

Again same procedure is done for the same object if it comes after some interval from the same camera view.

We add more steps in future extension of the project to enhance the more security and safe journey of peoples around the country.

\section{CONCLUSION}

Scene analysis and individual tracking through multiple camera network aim to enhance and track the security level of object that may include people with the help of background subtraction followed by individual tracking through multiple camera and then multiple individual tracking through multiple camera .There are many benefits of doing this in the field of computer vision as well as in security perspective. It includes firstly the scene analysis done using background subtraction and then the individual tracking done using the MST (mean shift tracking) and the enhancement of MST is done for doing the tracking through multiple camera that is tracking the individual object through multiple camera which is done by creating the unique bounding box along the objects.

\section{REFERENCES}

[1] "http://in.mathworks.com/products/matlab/"

[2] "https://en.wikipedia.org/wiki/MATLAB/"

[3] "http://in.mathworks.com/help/vision/ug/multiple-objecttracking.html"

[4] "http://in.mathworks.com/help/vision/examples/motionbased-multiple-object-tracking.html"

[5] "http://in.mathworks.com/help/vision/object-trackingand-motion-estimation.html",

[6] "http://in.mathworks.com/matlabcentral/answers/57299adaptive-background-subtraction-algorithm",

[7] "http://www.ics.uci.edu/ dramanan/teaching/cs117_sprin g11/lec/bg.pdf

[8] "http://in.mathworks.com/help/images/morphologicaldilation-and-erosion.html".

[9] "http://in.mathworks.com/help/vision/examples/usingkalman-filter-for-object-tracking.html

[10] "http://in.mathworks.com/help/vision/examples/motionbased-multiple-object-tracking.html

[11] "http://in.mathworks.com/matlabcentral/newsreader/view _thread/286289"

[12] "http://in.mathworks.com/matlabcentral/answers/38547masking-out-image-area-using-binary-mask"

[13] "http://in.mathworks.com/help/images/ref/imhist.html"

[14] "http://in.mathworks.com/help/images/create-imagehistogram.html"

[15] http://www.cse.psu.edu/ rtc12/CSE598G/introMeanShift .pdf"

[16] "http://www.serc.iisc.ernet.in/ venky/SE263/slides/MS_ Tracking.pdf”,

[17] "http://www.comp.nus.edu.sg/ cs4243/lecture/meanshift. pdf".

[18] "http://in.mathworks.com/matlabcentral/fileexchange/35 520-mean-shift-video-tracking”. 